\title{
CCV based image retrieval for FMIQ with confined histogram refinement
}

\author{
Satyawati M. Patil ${ }^{1}$, V.K.Patil ${ }^{2}$ \\ P.G. Student, E\&TC Department, D. N. Patel College of Engineering, Shahada, India ${ }^{1}$ \\ Professor \& H.O.D, E\&TC Department, D. N. Patel College of Engineering, Shahada, India ${ }^{2}$
}

\begin{abstract}
Content Based Visual Information Retrieval is one of the most cardinal and crucial aspect in the category of digital image processing. The significant mechanism that has been lucidly highlighted here is the fast multiresolution image querying using Color Coherence Vector $(\mathrm{CCV})$ along with the refinement of color histogram. However, the retrieval speed and accuracy can be enhanced using color characteristics and mathematical model despite the collection of innumerable images in dataset. Color feature extraction and coherency estimation assist in flawless and swiftest image querying according to the matched template (matrices) of the color histogram image with maximum precision.
\end{abstract}

Keywords: CCV, color histogram refinement, coherency image querying.

\section{INTRODUCTION}

With an ubiquity of growing standards in the field of scheme, as reported by P. S. Hiremath, S. Shivashankar, J. digital image processing, the necessity of easiest image Pujari,(2006) explains a similar kind of approach based on retrieval despite the collective library of several images color texture analysis by using different color spaces. has tremendously increased. Fast multiresolution image querying or retrieval basically deals with the searching of The work reported by Tian Yumin, Mei Lixia, "Image of desired images from the datasets on the basis of variety Retrieval Based on Multiple Features Using Wavelet," of color, texture, shape and sketch characteristics. This introduces a scheme based on wavelet which includes both paper highlights an extreme novel ideology of fast and color feature and texture feature circular region energy in flawless image retrieval from the magnificient database on low frequency band of wavelet transform of an image is the basis of Color Coherence Vector(CCV) generation used as color feature of the image and the synthesize along with the histogram refinement under the attributes energy in high frequency bands of multi-scale wavelet of the color characteristics and mathematical model for transforms is used as texture feature.

multiresolution approach.

However, due to maximum confinement of histogram features and the concerned strategies involved in the CCV computational mathematical model, image retrieval seems to possess extravagant potential in searching desired images with copious efficiency.

\section{BACKGROUND}

The concept of Content Based Image Retrieval (CBIR) was introduced by T. Kato in 1992.He initiated his experiments into automatic retrieval of images from a database on the basis of color and shape features. Furthermore, the database search and querying has received attention of several groups. Methods for reduction of the size of the characteristic vectors and increasing the speed of recovery in great databases were developed by [Swain-Ballard (1991)] and by [Stricker-Orengo (1995)]. Efficient index structures were developed by [Petrakis--Faloutsos (1995) and White--Jain (1996, 1997)].

\section{III.Proposed SYSTEM}

\section{A. System Model}

The significant mechanism that has been focused in this paper is the fastest image retrieval by generating color coherent vectors in addition to the combination of histogram feature extraction. Consequently,the error difference vectors are computed corresponding to the Euclidean distance and confined values stored in the database. Since CCV plays an active role in incrementing the speed of image retrieval,the query images are instantly retrieved at the required level.

Besides these, the registration process basically involves image acquisition, RGB to HSV conversion, segmentation, CCV computation and the computation of color coherency vectors. The proposed system strategy gets accomplished in the assortments of different stages.Figure 1 exhibits the main block diagram of proposed system which highlights the confined image retrieval using both techniques.

Pre-filter techniques that reduce the amount of images in database for similarity computation was accomplished by [Niblack et al. (1993)]. Several CBIR systems currently exist, and are being constantly developed. Another 


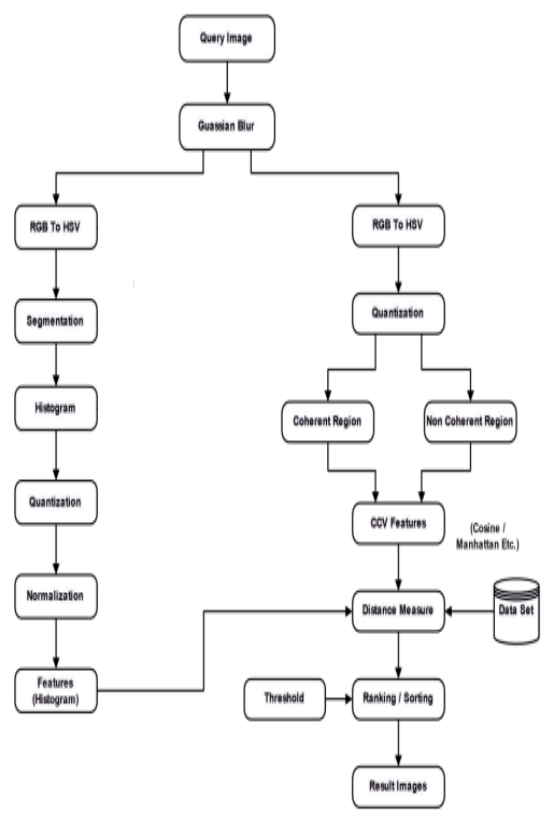

Fig.1 Combined Block Diagram of Color Histogram Refinement and $\mathrm{CCV}$

\section{B. Feature Extraction Strategies}

Content-based image retrieval, also known as query by image content or fast multiresolution image querying or content-based visual information retrieval is an application of computer vision to the image retrieval problem, that is, the problem of searching for digital images in large databases. Content-based means that the search makes use of the contents of the images themselves, rather than relying on human-input metadata such as captions or keywords. A content-based image retrieval system (CBIR) is a piece of software that implements CBIR. In CBIR, each image that is stored in the database has its features extracted and compared to the features of the query image. The key factor in the proposed scheme is of feature extraction at several instants and stages involved in CCV and color histogram mechanisms. Therefore, feature extraction for the several stages can be elaborated through algorithms. The first step in this process is to extract the image features to a distinguishable extent. In this paper, features are extracted to make system system more efficient and reliable.

\section{(a) Color Histogram Strategy}

The color histogram for an image is constructed by counting the number of pixels of each color. The approach more frequently adopted for CBIR systems is based on the conventional color histogram, which contains occurrences of each color obtained counting all image pixels having that color. Each pixel is associated to a specific histogram bin only on the basis of its own color, and color similarity across different bins or color dissimilarity in the same bin are not taken into account. Since any pixel in the image can be described by three components in a certain color space(for instance, red, green and blue components in RGB space or hue, saturation and value in HSV space), a histogram, i.e., the distribution of the number of pixels for each quantized bin, can be defined for each component.

By default the maximum number of bins one can obtain using the histogram function in MatLab is 256. The conventional color histogram $(\mathrm{CCH})$ of an image indicates the frequency of occurrence of every color in an image. The appealing aspect of the $\mathrm{CCH}$ is its simplicity and ease of computation. Once an input query image is acquired,blurring action is performed by Guassian filter.The blurred image undergoes the conversion from RGB to HSV color space and gets segmented into number of blocks. Comparing all the colors in two images would however be very time consuming and complex, and so a method of reducing the amount of information must be used. Oneway of doing this is by quantizing the colour distribution into colour histograms. While computing a colour histogram for an image, the different colour axes are divided into a number of so-called bins. A three dimensional 256x256x256 RGB histogram would therefore contain a total of 16777216 such bins. While indexing the image, the color of each pixel is found, and the corresponding bin's count is incremented by one. The normalized histogram can be obtained by dividing it with total number of pixels. Next to this is the computation of the histogram features and then store them as color feature vector. This computational scheme is performed for all the images present in the dataset. However,it seems to be easier to estimate Euclidean distance and error difference by comparing query image histograms to that of each image in the dataset. However, the dataset images are ranked and eventually sorted in ascending order of their mean difference query image and returned in the form of result.

\section{(b) Color Coherence Vector Strategy}

Intuitively, color coherency is the degree to which pixels of that color are members of large similarly-colored regions. Coherent pixels are a part of some sizable contiguous region, while incoherent pixels are not. A color coherence vector represents this classification for each color in the image. CCV's prevent coherent pixels in one image from matching incoherent pixels in another. This allows fine distinctions that cannot be made with color histograms. The initial stage in computing a CCV is similar to the computation of a color histogram. The image is blurred slightly by replacing pixel values with the average value in a small local neighborhood (generally including the 8 adjacent pixels). This eliminates small variations between neighboring pixels. However, the colorspace is discretized, in such a way that such that there are only $\mathrm{n}$ distinct colors in the image. The next step is to classify the pixels within a given color bucket as either coherent or incoherent. A coherent pixel is part of a large group of pixels of the same color, while an incoherent pixel is not. We determine the pixel groups by computing connected components. A connected component ' $C$ ' is a maximal set of pixels such that for any two pixels ' $p$ ' and ' $\mathrm{p}^{\wedge}$ ' corresponds to ' $\mathrm{C}$ ', there is a path in $\mathrm{C}$ between $\mathrm{p}$ and $\mathrm{p}^{\wedge}$. For a given discretized color, some of the pixels with that color will be coherent and some will be incoherent. 
Consider the number of coherent pixels of the j'th similarly colored images since content based image discretized color $\alpha \mathrm{j}$ and the number of incoherent pixels $\beta \mathrm{j}$ retrieval is not based on the exact matching. Suppose that . Clearly, the total number of pixels with that color is $\alpha \mathrm{j}$ ' $q$ ' is the query image while q' is the database image, then $+\beta \mathrm{j}$, and so a color histogram would summarize an image similarity measure can be evaluated by following steps: as $<\alpha 1+\beta 1, \ldots \ldots \alpha n+\_\beta n>$.Instead, for each color we Step1:.Determine color histogram vectors and CCV compute the pair $(\alpha \mathrm{j} ; \beta \mathrm{j})$ which we will call the coherence vectors of the database images.

pair for the $\mathrm{j}$ 'th color. The color coherence vector for the Step2:Compute color histogram vectors and CCV vectors image consists of $\langle(\alpha 1 ; \beta 1), \ldots \ldots .,(\alpha n ; \beta n)\rangle$. This is a of the query image.

vector of coherence pairs, one for each discretized color. Step3:Calculate Euclidean/Error difference distance Determination of 'tau's value. 'Tau' is a user-specified between two feature vectors.

value which is normally it's about $1 \%$ of image's Step4:If $\mathrm{d} \leq$ threshold, then images gets sorted or ranked size.With this value of 'tau' an average image in our and can be matched accordingly.

database consists of approximately $75 \%$ coherent pixels.

\section{C.Similarity Matching}

The second step involves matching these features to yield a result that is visually similar. Basic idea behind CBIR is that, when building an image database, feature vectors from images are to be extracted and then store the vectors in another database for future use. When given a query image its feature vectors are computed. If the distance between feature vectors of the query image and image in the database is small enough, the corresponding image in the database is to be considered as a match to the query. The search is usually based on similarity rather than on exact match and the retrieval results are then ranked accordingly to a similarity indexSimilarity measurement and matching occupies a crucial role in CBIR algorithms. These algorithms search image database to find images similar to a given query so that they should be able to evaluate the amount of similarities between the images. To measure the similarity, the direct Euclidean distance between the image in the database and the query image is given by,

$$
\text { E.D. }=\sum(\text { Vpi-Vqi })(V p i-V q i)
$$

where, Vpi and Vqi are the feature vectors of image and query image with respect to size'n'.It operates by considering each vector as a point in an ' $n$ ' dimensional vector space and assist in calculation of physical distance between two vector points of the images.

\section{- Matching function}

In $\mathrm{CCV}$,we compute two values ( $\mathrm{C}$ and $\mathrm{N}$ ) for each color; where ' $\mathrm{C}$ ' is the number of coherent pixels and ' $\mathrm{N}$ ' is the number of incoherent pixels.It is clear that the summation of all color's $\mathrm{C}$ and $\mathrm{N}=$ number of pixels. Suppose that we have to compare 2 images a, b. Therefore, matching function can be elaborated as,

$$
\boldsymbol{D}(\boldsymbol{a}, \boldsymbol{b})=\sum_{i=\mathbf{0}}^{n} \quad\left(\left|\mathbf{a}_{\mathrm{Ci}}-\mathbf{b}_{\mathrm{Ni}}\right|\right)+\left(\left|\mathbf{a}_{\mathrm{Ni}}-\mathbf{b}_{\mathrm{Ni}}\right|\right)
$$

' $\mathrm{C}$ ' ${ }_{i \text {. }}$ represents number of coherent pixels in color ' $i$ ' and ' $\mathrm{N}$ ' ${ }_{i}$ represents number of incoherent pixels in color ' $\mathrm{i}$ '.

This algorithm implies the strategy of matching on color by color basis. Inceptionally, the number of colors in query image and database image are estimated by analyzing histogram .However, if the amount of specific color in both the images are comparable, then the images gets easily matched. Retrieval result is not based on a display of a single image but the combination of multiple

\section{Generation of Image Database}

This system comprises the database of about several images including different categories with the assortments of several images. All the images are stored in jpg format of different dimension sizes. The image data used in the experiment were taken from wang and corel databases to some extent. However, the modules and tools of the system can be incremented as per the required level.However in order to reduce the computation time of the whole process,image sizes can also be reduced to $8 \times 8$ pixels.

\section{IV.EXPERIMENTAL ANALYSIS AND RESULTS}

Generally, there are two parameters namely precision and recall which helps to evaluate the performance of image retrieval system. For a query q, the data set of images in the database that are relevant to the query $\mathrm{q}$ is denoted as $R(q)$, and the retrieval result of the query $\mathrm{q}$ is denoted as $Q(q)$.It has been reported that the histogram exhibits the best performance through the estimation of following parameters.

(a)Precision: The precision of the retrieval is defined as the fraction of the retrieved images that are indeed relevant for the query. It measures the ability of the system to retrieve only the models that are relevant. It can be defined as,

Precision $=\frac{\text { Number of relevant images retrieved }}{\text { Total number of images retrieved }}$

(b) Recall: The recall can be defined as the fraction of relevant images that is returned by the query.It measures the ability of the system to retrieve all the models that are relevant.

Recall $=\frac{\text { Number of relevant images retrieved }}{\text { Total number of relevant images }}$

(c) Accuracy: An accuracy of the system can be defined as the half the fraction sum of precision and recall values, which can be expressed as, Accuracy $=($ Precision + Recall $) / 2$

(d) Match Percentile: The match percentile MP of a given image is expressed as:

$$
\mathbf{M P}=(\mathbf{N}-\mathbf{R}) /(\mathbf{N}-\mathbf{1})
$$


INTERNATIONAL JOURNAL OF INNOVATIVE RESEARCH IN ELECTRICAL, ELECTRONICS, INSTRUMENTATION AND CONTROL ENGINEERING Vol. 3, Issue 1, January 2015

where $\mathrm{N}$ is the number of images in the database and $\mathrm{R}$ is the rank of the returned image. All these parameters are calculated for each image and the results are averaged.Our system has been implemented to execute on the platform of MATLAB R2010 'a' version along with the procession of several modules related to digital image processing. It utilizes several tools of MATLAB concerned with the image acquisition, preprocessing, segmentation, quantization, segmentation, thresholding and so on. This system utilizes the database of

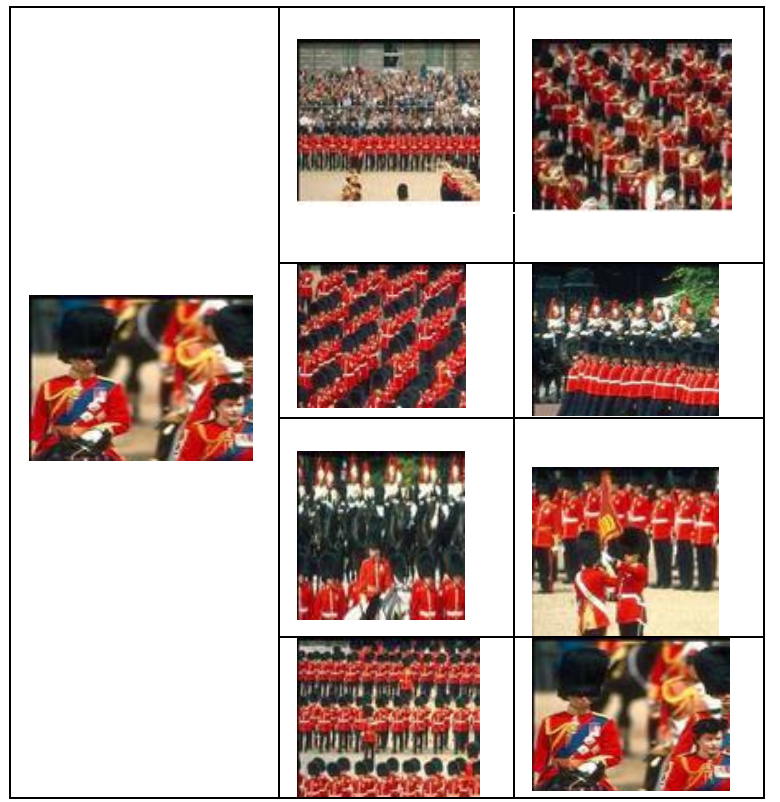

Fig.2. A sample of Query image and the retrieved similar images by proposed method

Table1 indicates an ideology of computed precise experimental results of color histogram refinement mechanism. The tabulated values are averaged for each and every present in the category of query images so as to estimate precision and recall values. Precision and recall measures the accuracy confined match percentile of image retrieval efficiency of the overall system.

Table 1. Retrieval Results of CHR Mechanism

\begin{tabular}{|l|l|l|l|l|}
\hline \multirow{2}{*}{$\begin{array}{l}\text { Query } \\
\text { Image } \\
\text { Categories }\end{array}$} & \multicolumn{4}{|c|}{$\begin{array}{l}\text { Color Histogram } \\
\text { Refinement Technique }\end{array}$} \\
\cline { 2 - 5 } & $\begin{array}{l}\text { Precision } \\
\text { Rate } \\
{[\%]}\end{array}$ & $\begin{array}{l}\text { Recall } \\
\text { Rate } \\
{[\%]}\end{array}$ & $\begin{array}{l}\text { Accuracy } \\
\text { Rate } \\
{[\%]}\end{array}$ & $\begin{array}{l}\text { Match } \\
\text { Percentile } \\
{[\%]}\end{array}$ \\
\hline $\begin{array}{l}\text { British } \\
\text { Army }\end{array}$ & $\mathbf{8 3 . 2}$ & $\mathbf{7 2 . 2}$ & 77.7 & 71.8 \\
\hline Mountain & 94.3 & 60.4 & 77.3 & 68.6 \\
\hline Bear & 91.5 & 80.1 & 85.9 & 80.2 \\
\hline Deer & 58.5 & 55.6 & 56.5 & 52.4 \\
\hline Eagle & 54.2 & 51.3 & 52.5 & 49.2 \\
\hline Firework & 65.8 & 55.2 & 60.4 & 57.1 \\
\hline \multicolumn{1}{|c|}{ AVG } & 74.5 & 62.4 & 68.3 & 63.2 \\
\hline
\end{tabular}

With the advent of several search engines, image searching has almost become an easier task. But, the existing search engines utilizes textbased image indexing and retrieval mechanisms which are not sufficient to the upheaval requirement. Therefore, fast multiresolution image querying provides an extravagant impact to complement existing mechanisms so as to allow the system to retrieve more similar images from the source images and hence provide effectively precise results. The major objective of this paper signifies the strategy of improvement in the accuracy and speed of image retrieval in order to handle predicaments of large image databases while optimizing several applications with more efficiency.

\begin{tabular}{|l|l|l|c|c|}
\hline \multirow{2}{*}{$\begin{array}{l}\text { Query } \\
\text { Image }\end{array}$} & \multicolumn{4}{|c|}{ Color Coherence Vector Technique (CCV) } \\
\cline { 2 - 5 } & $\begin{array}{l}\text { Precision } \\
\text { Rate } \\
{[\%]}\end{array}$ & $\begin{array}{l}\text { Recall } \\
\text { Rate } \\
{[\%]}\end{array}$ & $\begin{array}{l}\text { Accuracy } \\
\text { Rate } \\
{[\%]}\end{array}$ & $\begin{array}{l}\text { Match } \\
\text { Percentile } \\
{[\%]}\end{array}$ \\
\hline $\begin{array}{l}\text { British } \\
\text { Army }\end{array}$ & 98.5 & 93.7 & 96.1 & 92.9 \\
\hline Mountain & 99.9 & 85.9 & 92.9 & 89.8 \\
\hline Bear & 96.8 & $\mathbf{8 3 . 5}$ & 90.1 & 83.6 \\
\hline Deer & 60.4 & $\mathbf{8 0 . 6}$ & 70.5 & 75.4 \\
\hline Eagle & 95.3 & 40.8 & 68.0 & 72.5 \\
\hline Firework & 97.6 & 86.9 & 92.2 & 78.9 \\
\hline AVG & 91.4 & 78.5 & 84.9 & 81.6 \\
\hline
\end{tabular}

Table 2 exhibits the image retrieval estimated results retrieval efficiency can also indexed in terms of several parameters as displayed in Fig.3. Both the methods yields ptimum lucid and accurate results since both utilizes the color feature for image retrieval purpose but $\mathrm{CCV}$ offers maximum proficiency on comparison basis.

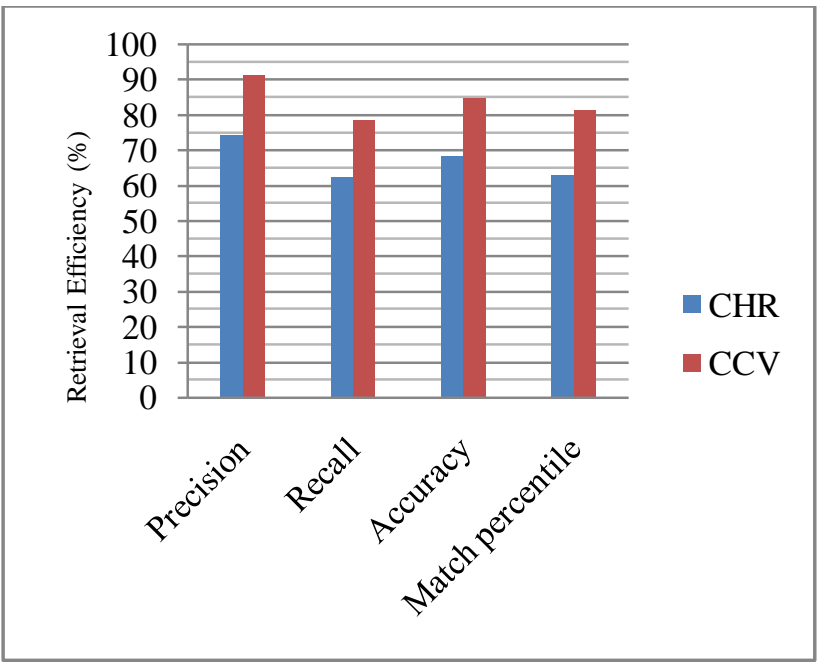

Figure 3. Comparative Color Histogram Refinement and Color efficiency measurement parameters

\section{V.CONCLUSION}




\section{REFERENCES}

[1] K.Valli Madhav1, R.Tamilkod, .Bala Dinakar, K.JayaSudha, "An Innovative Technique for Content Based Image Retrieval Using Color and Texture Features," International Journal of Innovative Research in Computer and Communication Engineering,Vol. 1, Issue 5, July 2013.

[2] G.Raghupati, R.S.Anandand M.L.Dewal, "color and texture features for content based image retrieval using motif", second international conference on multimedia and content based image retrieval, july21-23,2010.

[3] Young Deok Chun, Nam Chul Kim, Member, IEEE, and Ick Hoon Jang, Member, IEEE, "Content-Based Image Retrieval Using Multiresolution Color and Texture Features", IEEE Transactions On Multimedia, Vol. 10, No. 6, October 2008.

[4] Michael S. Lew, Nicu Sebe, Chabane Djeraba, and Ramesh Jain, "Content-based multimedia information retrieval: State of the artand challenges," ACM Trans. Multimedia Comput. Commun. Appl., vol. 2, no. 1, pp. 1-19, 2006.

[5] Irena Valova, Boris Rachev and Michael Vassilakopoulos, "Optimization of the Algorithm for Image Retrieval by Color Features",International Conference on Computer Systems and Technologies- CompSysTech"e, pp 1-4, 2006.

[6] Shamik Sural, Gang Qian and Sakti Pramanik, "Segmentation and Histogram Generation Using the HSV Color Space for ImageRetrieval", International Conference on Image processing, Vol. 2, pp. 589-592, 2002.

[7] Aleksandra Mojsilovic, Jianying $\mathrm{Hu}$ and Emina Soljanin, "Extraction of Perceptually Important Colors and Similarity Measurement for Image Matching, Retrieval, and Analysis", IEEE Transactions on Image Processing, Vol. 11, No. 11, pp 1238-1248, November 2002.

[8] Greg Pass, Ramin Zabih and Justin Miller, "Comparing Image Using Color Coherence Vectors", Proceedings of the fourth ACM international conference on Multimedia,pp.65-73,1996.

[9] Vellaikal and C. C. J. Kuo, Content Based Image Retrieval using Multiresolution Histogram Representation "ee, SPIE - Digital Image Storage and Archiving Systems, Vol. 2606, pp. 312-323, 1995 